\title{
Interaction of terahertz electromagnetic field with a metallic grating: Near-field zone
}

\author{
Lyaschuk Yu. M. and Korotyeyev V. V. \\ Department of Theoretical Physics, V. Lashkaryov Institute of Semiconductor Phys- \\ ics, National Academy of Sciences of Ukraine, 01650 Kyiv, Ukraine, \\ yulashchuk@gmail.com, koroteev@ukr.net
}

Received: 14.05 .2012

\begin{abstract}
We have developed a theory for the interaction of $\mathrm{THz}$ radiation with a sub-wavelength metallic grating. The structure of electric field of the electromagnetic waves under the metallic grating has been studied for the near-field zone. Spatial distributions of the electric field components and the electric energy density have been obtained for the wave transmitted through the grating. An effect of strong local enhancement of the electric field has been detected. Spatial dependence of polarisation of the transmitted wave has been analysed for the near-field zone.
\end{abstract}

Keywords: terahertz radiation, near-field optics, plasmonics

PACS: $85.60 .-\mathrm{q}, 07.57-\mathrm{c}$, 42.25.Bs, 42.79.Pw

UDC: $537.874,537.862$

\section{Introduction}

In recent years, one of the priority directions in development of modern nanoelectronics and optoelectronics is elaboration of solid-state sources, detectors and modulators of electromagnetic (EM) waves in terahertz (THz) spectral range. These researches have been inspired by numerous potential applications of $\mathrm{THz}$ optics, including communication technologies, wireless local area networks, spectral analysis of complex molecules and materials, time-domain spectroscopy, $\mathrm{THz}$ imaging (in particular, for medical applications), etc. [1, 2].

One of the most attractive directions in the development of $\mathrm{THz}$ optoelectronics is $\mathrm{THz}$ plasmonics that investigates controlling of the corresponding radiation by using excitation of plasma oscillations (plasmons) in semiconductor microdevices. During the past decade, a lot of theoretical and experimental works have been devoted to the problems of detecting and emitting the $\mathrm{THz}$ radiation by means of excitation of plasma waves in 2D channel of field-effect transistors [3, 4]. Here better operation conditions have been achieved for the transistors with multi-gated structures. A periodic system of metallic gates (i.e., a metallic grating) plays a role of specific antenna element that provides efficient coupling of relatively long-wavelength THz-range EM-radiation with short-wavelength plasmons. Resonant detection and amplification of the $\mathrm{THz}$ radiation have recently been studied for the multi-gated field-effect transistors [5-8]. Moreover, similar multicontact devices can be utilised as modulators and polarisers for the THz radiation $[9,10]$. In the experiments with transmission of the $\mathrm{THz}$ radiation one typically uses structures with a metallic grating of a sub-wavelength period $(a)$ and a submicron distance $(D)$ between the grating and a 2D electron gas. Such a geometry is preferable for efficient excitation of plasmons with $\mathrm{THz}$ fre- 
quencies. The interaction of EM-waves with plasmons in these structures occurs in a near-field zone $(D<<a)$ near the metallic gratings, where the properties of the EM-fields are very different from those peculiar for the far-field zone.

The present article addresses a detailed analysis of structure of the EM-field transmitted through the single metallic grating in the near-field zone. In Section 2 we describe a theoretical approach employed for solving the problem of interaction of the EM-waves with the periodic metallic gratings. The results of calculations for the spatial distribution of the EM-field located under the grating and its polarisation characteristics are discussed in Sections 3 and 4. Finally, the conclusions are drawn in Section 5.

\section{Theory of interaction of light with metallic gratings}

A typical geometry of structure of the grating is shown in Fig. 1. The grating consists of metallic strips, with the width $b$ and the thickness $d$, which are arranged along the $x$ direction with the period $a$. The system is assumed to be uniform and infinite along the $y$ direction. Let a plane monochromatic EM-wave be incident upon the grating along the $z$ axis, with the electric vector polarised in the $x$ direction (i.e., perpendicular to the grating strips). We consider the case of a subwavelength grating $\left(a<\lambda_{0}\right)$, with $\lambda_{0}$ denoting the wavelength of the incident EM-wave. The grating strips will be treated as conducting layers infinitely thin along the $z$ direction. This assumption is justified whenever a skin layer is much thicker than $d$ (e.g., the estimations for the gold strips yield in the skin layer thickness of about $80 \mathrm{~nm}$ at $1 \mathrm{THz}$ ). In order to satisfy the assumption mentioned above, all of our calculations will be carried out for the thickness of $d=20 \mathrm{~nm}$. Finally, the grating system is considered as being placed into uniform environment with the dielectric constant $\varepsilon$.

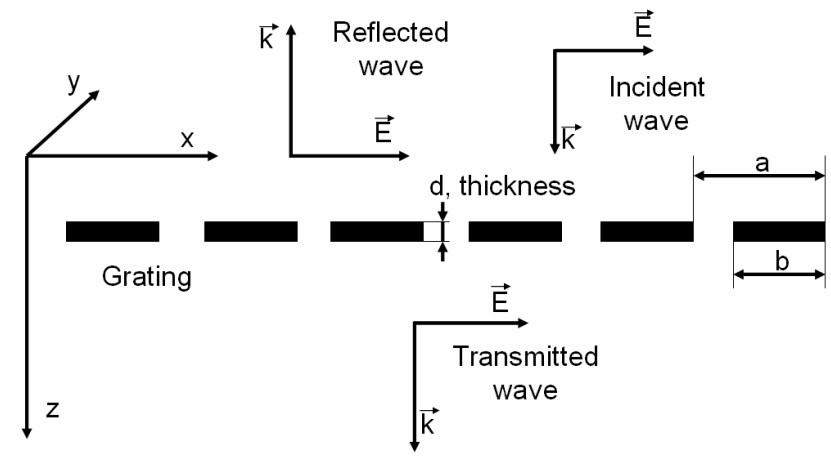

Fig. 1. Illustration of geometry of a metallic grating.

Being a result of interaction of the plane wave with the metallic grating, the total electric field obeys the Maxwell equation,

$$
\operatorname{rot} \operatorname{rot} \vec{E}(\vec{r}, t)+\frac{\varepsilon}{c^{2}} \frac{\partial^{2} \vec{E}(\vec{r}, t)}{\partial t^{2}}=-\frac{4 \pi}{c^{2}} \frac{\partial \vec{j}(\vec{r}, t)}{\partial t} .
$$

In Eq. (1), $\vec{j}(r, t)$ is the conduction current induced by the EM-field in the metallic strips. Due to symmetry of the problem, the electric field $\left(\vec{E}(r, t)=\left\{E_{x}(x, z), 0, E_{z}(x, z)\right\} \exp (-i \omega t)\right)$ and the current $\left(\vec{j}(r, t)=\left\{j_{x}^{2 D}(x) \delta(z), 0,0\right\} \exp (-i \omega t)\right)$ have two components and one compo- 
nent, respectively. Then the vector equation given by formula (1) may be rewritten as a system of two scalar equations:

$$
\begin{aligned}
& \frac{\partial^{2} E_{z}(x, z)}{\partial x \partial z}-\frac{\partial^{2} E_{x}(x, z)}{\partial^{2} z}-\omega^{2} \frac{\varepsilon}{c^{2}} E_{x}=\frac{4 \pi i \omega}{c^{2}} j_{x}^{2 D}(x) \delta(z), \\
& \frac{\partial^{2} E_{x}(x, z)}{\partial x \partial z}-\frac{\partial^{2} E_{z}(x, z)}{\partial^{2} x}-\omega^{2} \frac{\varepsilon}{c^{2}} E_{z}=0 .
\end{aligned}
$$

According to the Ohm law, the current $j_{x}(x)$ in the strips may be expressed in terms of the total electric field in the plane $z=0$ :

$$
j_{x}^{2 D}(x)=\sigma^{2 D}(x) E_{x}(x, 0),
$$

Iwith $\sigma^{2 D}(x)$ being the local 2D conductivity of the metallic strips. Structural periodicity of the grating along the $x$ direction allows one searching for the solution of the system of Eqs. (2) in the form of Fourier series expansion:

$$
E_{(x, z)}(x, z)=\sum_{m=-\infty}^{+\infty}\left\{E_{x, m}^{i}(z)+E_{(x, z), m}^{s}(z)\right\} e^{i q} m^{x},
$$

where $q_{m}$ is the wave number of the grating $\left(q_{m}=2 \pi m / a\right)$. Eq. (4) describes the total electric field as a sum of two contributions. The first one, $E_{x}^{i}(z)$, describes the external incident field. We choose this field to be a plane monochromatic wave $\left(E_{x, m}^{i}(z)=E^{i} \delta_{m, 0} e^{i k_{0} z}\right.$, with $\left.k_{0}=\sqrt{\varepsilon} \omega / c\right)$. The second contribution, $E_{(x, z)}^{s}(z)$, is the scattered field which is a result of re-emission induced by the alternating current produced in the metallic strips.

After inserting Eq. (4) into Eqs. (2), we obtain the equation for the $m$ th Fourier coefficient of the $x$-component of the scattered field $E_{x, m}^{s}$,

$$
\frac{\partial^{2} E_{x, m}^{s}}{\partial z^{2}}-\lambda_{m}^{2} E_{x, m}^{s}=\frac{4 \pi i \lambda_{m}^{2}}{\varepsilon \omega} j_{x, m}^{2 D},
$$

along with the relationship between the $m$ th Fourier coefficients for the $x$ - and $z$-components:

$$
E_{z, m}^{s}=-i \frac{q_{m}}{\lambda_{m}^{2}} \frac{\partial E_{x, m}^{s}}{\partial z}
$$

Eq. (5) has the solutions

$$
E_{m, x}^{S}(z)=A_{m} e^{\lambda m^{z}} \text { at } z<0 \text { and } E_{m, x}^{S}(z)=B_{m} e^{-\lambda m^{z}} \text { at } z>0,
$$

where $\lambda_{m}=\sqrt{q_{m}^{2}-k_{0}^{2}}$. When $m=0$, we have $\lambda_{m}=-i k_{0}$ (here the sign minus is chosen such that the solutions would give radiating modes) and the constants $A_{0}$ and $B_{0}+E^{i}$ are amplitudes respectively of the reflected wave and the transmitted wave in the far-field zone.

In the case of sub-wavelength grating, $\lambda_{m}$ becomes purely real at $m \neq 0$ and so the constants $A_{m}$ and $B_{m}$ represent the amplitudes of evanescent waves. The evanescent waves are localised near the grating and their intensity decreases exponentially with increasing distance from the grating. Due to translation symmetry of the grating along the $x$ direction, the evanescent waves are equivalent for $q_{m}$ and $q_{-m}$. Therefore, the EM-field in the near-field zone is nothing but a stand- 
ing wave. The unknown constants $A_{m}$ and $B_{m}$ may be found from the following boundary conditions valid for the plane $z=0$ :

$$
\left.E_{x, m}^{s}(z)\right|_{z=+0}=\left.E_{x, m}^{s}(z)\right|_{z=-0},\left.\frac{\partial E_{x, m}^{s}(z)}{\partial z}\right|_{z=+0}-\left.\frac{\partial E_{x, m}^{s}(z)}{\partial z}\right|_{z=-0}=\frac{4 \pi i \lambda_{m}^{2}}{\omega \varepsilon} j_{x, m}^{2 D} .
$$

The conditions given by Eqs. (8) imply that $A_{m}=B_{m}$ and $B_{m}=-2 \pi i \lambda_{m} j_{x, m}^{2 D} / \omega \varepsilon$. Using the Ohm law (see Eq. (3)), one can express the $m$ th Fourier coefficient of the current as a convolution product of the Fourier components of the $2 \mathrm{D}$ conductivity and the total electric field calculated at $z=0$ :

$$
j_{x, m}^{2 D}=\left.\sum_{m^{\prime}=-\infty}^{\infty} \sigma_{m-m^{\prime}}^{2 D} E_{x, m^{\prime}}\right|_{z=0} .
$$

Taking into account that $B_{m}+E^{i} \delta_{m, 0}=\left.E_{x, m}\right|_{z=0}$, we get

$$
\left.\sum_{m^{\prime}=-\infty}^{\infty}\left\{\delta_{m, m^{\prime}}+\frac{2 \pi i \lambda_{m}}{\varepsilon \omega} \sigma_{m-m^{\prime}}^{2 D}\right\} E_{x, m^{\prime}}\right|_{z=0}=E^{i} \delta_{m, 0} .
$$

Thus, we have reduced the electrodynamic problem of light interaction with the periodic metallic grating to an infinite system of algebraic equations. After solving numerically Eqs. (10), with large though finite number $M$ of the equations, one can obtain spatial distribution of all the components of the electric field vector of the transmitted wave:

$$
E_{x}(x, z)=\left.\sum_{m=-M}^{M} E_{x, m}\right|_{z=0} e^{-\lambda_{m} z} e^{i q_{m} x}, E_{z}(x, z)=\left.\sum_{m=-M}^{M} \frac{i q_{m}}{\lambda_{m}} E_{x, m}\right|_{z=0} e^{-\lambda_{m} z} e^{i q_{m} x} .
$$

Good enough convergence of the solutions given by Eqs. (11) with increasing number $M$ can be achieved while assuming that the metallic strip is described by some smoothed conductivity profile. In the present work, we make use of the profile defined by

$$
\sigma^{2 D}(x)=\left\{\sigma_{0}^{2 D} \sin ^{p}(\pi x / b), x \in[0, b] \text { and } 0, x \in[b, a]\right\},
$$

where $p$ means a fractional number. This profile is convenient because its Fourier coefficients have the analytical form:

$$
\sigma_{m}^{2 D}=\sigma_{0}^{2 D} f 2^{1-p}(p+1)^{-1} \exp (-i \pi m f) B^{-1}(p / 2+m f+1, p / 2-m f+1),
$$

with $f=b / a$ being the filling factor of the grating and $B$ the Euler beta-function.

Notice that another analytical approach to the grating-related problems is often used in the literature. In frame of this method, the system of Eqs. (10) is reformulated in terms of an integral equation in the coordinate space. The general scheme of its solution is based on expansion of the total field in a series of polynomials orthogonal with respect to the weight function $\sigma^{2 D}(x)$. This procedure is again reduced to an infinite set of equations for the expansion coefficients. The approximate solution of the latter has somewhat better convergence when compared with our system (see Eqs. (10)). However, such a procedure can only be applied for specific profile shapes $\sigma^{2 D}(x)$. For instance, the authors of Ref. [11] have analysed plasma eigenmodes for the system consisting of a grating and a 2D electron gas, using a semi-elliptic profile of the strip conductivity. Meanwhile, our method is applicable for arbitrary profiles $\sigma^{2 D}(x)$. Specific results obtained while solving directly Eqs. (10) are described in the next section. 


\section{Properties of the electric field located under the grating}

In Fig. 2 we illustrate instantaneous spatial distributions of the $x$ - and $z$-components of the total electric field $\operatorname{Re}\left[E_{x, z}(x, z) e^{-i \omega t}\right]$ of the wave transmitted below the grating. The coordinates $x \in[0,0.5 a]$ and $x \in[0.5 a, a]$ correspond to the location of the metallic strip and the window between the strips, respectively. As seen from Fig. 2, the electric field has essentially non-uniform distribution in the near-field zone (see curves 1 and 2). This is a result of complicated superposition of the incident and scattered fields, the latter being induced by redistribution of charge in the metallic strips. Notice also that the scattered field is a superposition of the evanescent waves.
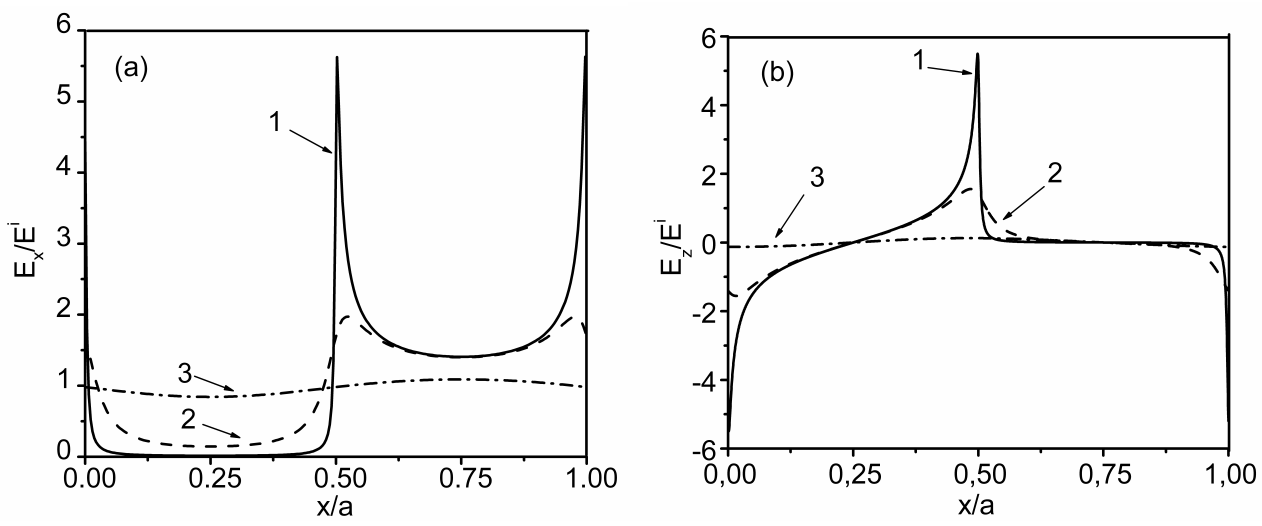

Fig. 2. Instantaneous spatial distributions of fields $E_{x, z}(x, z, t)$ at $t=0:$ panels (a) and (b) correspond respectively to the $E_{x}$ and $E_{z}$ components, and curves 1,2 and 3 are calculated at $z / a=0.003,0.03$ and 0.3 , respectively. The grating period is equal to $a=30 \mu \mathrm{m}$, the filling factor $f=0.5$, the strip conductivity is chosen as for the gold $\left(\sigma_{0}^{2 D}=8 \times 10^{12} \mathrm{~cm} / \mathrm{s}\right)$, the strip thickness $d=20 \mathrm{~nm}$, the fractional number $p=1 / 6$, the frequency of the incident wave $\omega / 2 \pi=1 \mathrm{THz}$, and $\varepsilon=1$.

One can observe a strong enhancement of the electric field near the edges of the metallic strips. Both the $x$ - and $z$-components exist in this narrow region and, moreover, each of them has the values much greater than the amplitude of the incident wave. It is worthwhile that the $z$ component is equal to zero at the point $x=0.25 a$ (i.e., in middle of the strip). This fact indicates that the spatial distribution of the scattered field is similar to that typical for the field of a dipole, with the corresponding electric charges induced on the opposite edges of the strip. The dipole has such an instantaneous polarity that its field almost totally screens the incident field under the strip. It is clearly seen from Fig. 2 that the total field has a very small $x$-component of the electric field in the main region under the metallic strip, though the $z$-component remains non-vanishing, except for in the very middle of the strip.

However, we observe the opposite situation under the window (except for the edge regions). Here the incident field is not screened, but rather amplified by the scattered field, so that the former field mainly contributes to the total one. The total field has a vanishing $z$-component and a non-vanishing $x$-component, which remains practically uniform. The values of the $x$-component of the electric field are slightly larger than the amplitude of the incident wave. The spatial distribution of the total electric field becomes more and more uniform with increasing distance from the grating, since the contribution of the evanescent modes which have formed the scattered field de- 
creases exponentially. At the distance $z \sim a$ (see curve 3 in Fig. 2) we deal with the case of farfield zone, where the main contribution to the transmitted wave originates from the mode with $m=0$.

The distribution of the electric field in the near-field zone also depends on the grating parameters. As shown in Fig. 3, the effect of enhancement of the electric field is more pronounced for the gratings with larger filling factors (i.e., narrower windows). For the case of $f=0.8$ (see curve 2 in Fig. 3), the field distribution under the window is stipulated by the charges induced at the right and left edges of the adjacent strips. Obviously, decreasing distance between these charges leads to increasing amplitudes of the electric fields.
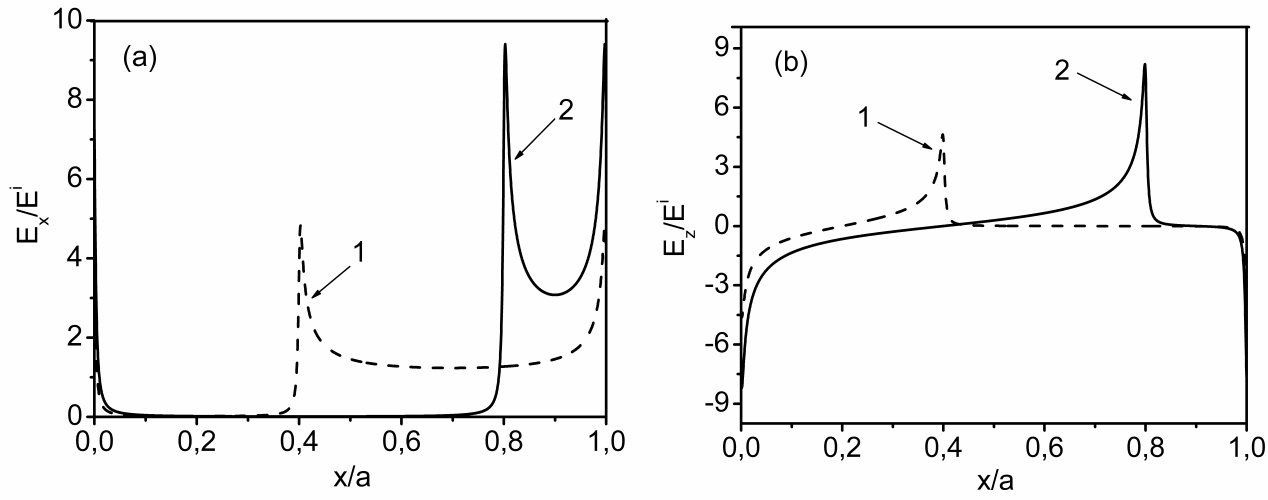

Fig. 3. Instantaneous spatial distributions of components $E_{x}$ (a) and $E_{z}$ (b) of the electric field transmitted under the grating: curves 1 and 2 are calculated for the filling factors $f=0.4$ and $f=0.8$, respectively. The calculations have been performed for $z / a=0.0033(z=0.1 \mu \mathrm{m})$ and the other parameters are the same as in Fig. 2.

In order to clarify the major features of the near-field zone, Fig. 4 depicts spatial mapping of the normalised time-averaged density of the electric energy, $W(x, z)$, of the transmitted wave. This quantity is calculated as $W(x, z)=\left(\left|E_{x}(x, z)\right|^{2}+\left|E_{z}(x, z)\right|^{2}\right) / E^{i 2}$, where $E_{x}(x, z), E_{z}(x, z)$ are given by Eqs. (11).
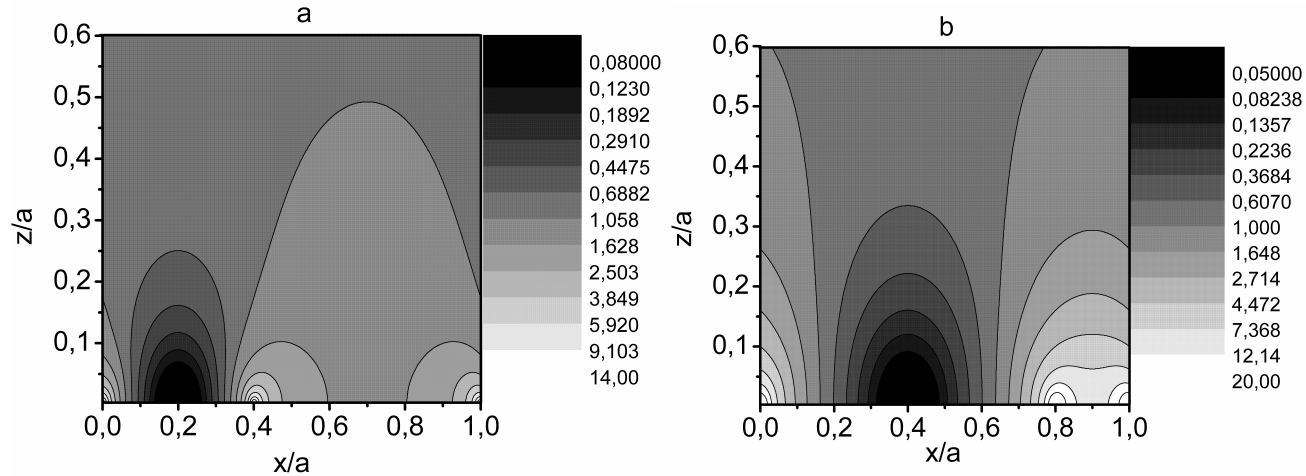

Fig. 4. Counter plots of normalised time-averaged electric energy density $W(x, z)$ of the transmitted wave calculated for the filling factors $f=0.4$ (a) and $f=0.8$ (b). All the other parameters are the same as in Fig. 2. Notice that the parameter $W(x, z)$ has been normalised to the corresponding value for the incident wave. 
The metallic grating produces a strong spatial redistribution of the energy of the (initially uniform) incident wave. Fig. 4 clearly demonstrates the existence of several zones with different energy concentrations. For relatively small distances from the grating, the energy of the transmitted wave is mainly concentrated near the edges of the strip. This corresponds to three hot zones (see white regions in Fig. 4). A cold zone is formed in the region under the metallic strip (see black region in Fig. 4). The lowest energy concentration corresponds to the middle of the strip where both the $x$ - and $z$-components of the field are close to zero. While moving away from the grating, the EM-wave penetrates under the metallic strip and the energy is transferred from the hot zones to the cold one. An almost uniform distribution of the $W(x, z)$ parameter is established at the distances from the grating plane as large as $z \sim a / 2$ (see Fig. 4a). When the grating has a structure with larger filling factors (see Fig. 4b), we get stronger redistribution of the energy and therefore the near-field zone becomes broader.

Nowadays, there is a number of experimental techniques that allow obtaining object images beyond the diffraction limit, using special probes with sub-wavelength apertures [12]. Then a planar object scanned in front of this aperture could be imaged with a resolution determined by the aperture size rather than the radiation wavelength. A high-resolution technique for terahertz nearfield imaging, which employs a planar structure with a single sub-wavelength aperture and 2D electron gas as a detector, has recently been reported in Ref. [13]. In principle, the similar methods may be applied while visualising the $\mathrm{THz}$ fields in the near-field zone of the periodic metallic structures.

\section{Polarisation of the electromagnetic wave in the near-field zone}

As already mentioned above, the real vector of the total electric field in the near-field zone has the two components $E_{x, z}(x, z, t)=\operatorname{Re}\left[E_{x, z}(x, z) e^{-i \omega t}\right]$. During one oscillation period, a terminus of the local electric field vector will circumscribe some polarisation ellipse in the $\left\{E_{x}, E_{z}\right\}$ plane. The ellipticity $\delta$ and the azimuth angle $\alpha$ of the polarisation ellipse may be expressed as follows:

$$
\delta=\left[\left(1-\sqrt{1-\beta^{2}}\right) /\left(1+\sqrt{1-\beta^{2}}\right)\right]^{1 / 2}, \tan \alpha=\left[2 r^{2}-\left(1+r^{2}\right)\left(1-\sqrt{1-\beta^{2}}\right)\right] / 2 \operatorname{rcos}(\Delta \phi),
$$

where $\beta=2 r \sin (\Delta \varphi) /\left(1+r^{2}\right), r=\left|E_{z}(x, z)\right| /\left|E_{x}(x, z)\right|$, and $\Delta \phi$ denotes the phase shift between the components $E_{x}(x, z, t)$ and $E_{z}(x, z, t)$. It is evident that these two polarisation parameters would depend upon the local point $(x, z)$.

The left panel of Fig. 5 illustrates the local polarisation ellipses observed at a fixed coordinate $z$ for different $x$ coordinates. They correspond to the regions under the strip, near the edge of the strip, and under the window. The right panel of Fig. 5 depicts evolution of the polarisation ellipses with increasing distance from the grating at a fixed coordinate $x$. Both the $z$ - and $x$-components tend to zero very close to the middle of the strip and the polarisation ellipse degenerates into a point. While moving towards the edge of the strip, the $z$-component rapidly increases though the $x$ component remains small ( $r>>1$ ). Thus, we observe increase in both the length of the major axis of the ellipse and the azimuth angle $\alpha$ (see curves 1 and 2 in Fig. 5a). Large parameters $r$ also induce small ellipticities of the polarisation ellipses. Near the edge of the strip (see curve 3 in Fig. 5a), the $x$ - and $z$-components oscillate practically in-phase ( $\Delta \phi \sim 0)$, with very close amplitudes $(r \sim 1)$. As a consequence, the polarisation ellipse degenerates into a straight line, with 
$\alpha \sim 45^{\circ}$. In the region under the window $(r<<1)$, we have the ellipse with a small azimuth angle and, again, a small ellipticity (see curve 4 in Fig. 5). The parameter $r$ decreases with increasing distance from the grating and we observe a monotonic decrease in the $\alpha$ parameter (see Fig. 5b). The polarisation ellipses degenerate into horizontal straight lines far from the grating, so that the transmitted EM-wave becomes linearly polarised.
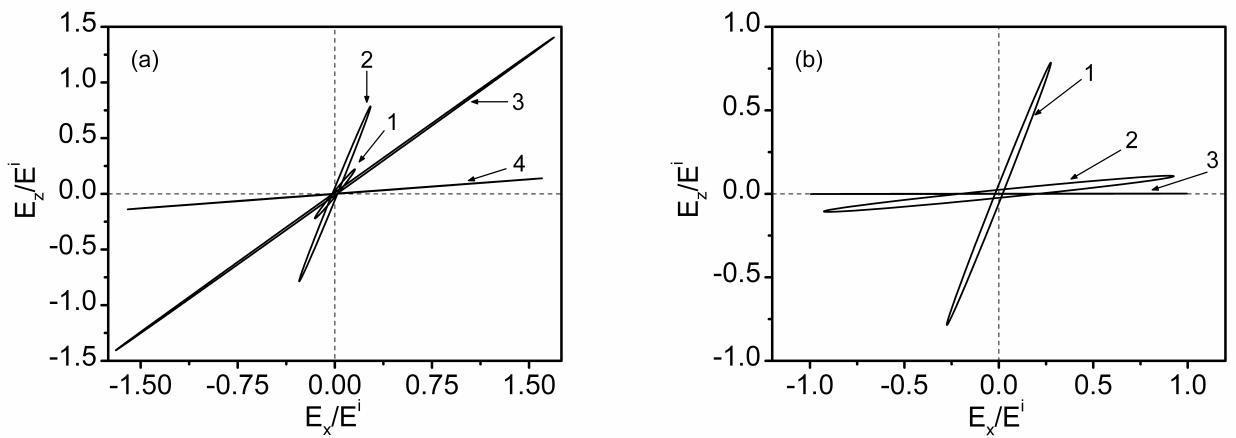

Fig. 5. Local polarization ellipses for (a) $z / a=0.033, z=1 \mu \mathrm{m}$ (curves $1,2,3$ and 4 correspond to $x / a=0.3,0.4,0.5$ and 0.6 , respectively) and (b) $x / a=0.4$ (curves $1,2,3$ correspond to $z / a=0.03,0.3$ and 1, respectively). The other parameters are taken the same as in Fig. 2.

Summarising the results obtained in this section, we note that the metallic grating produces a complicated wave field in the near-field zone, which is characterised with strong spatial dependences of both the local energy and the polarisation characteristics. Such a property in the near-field can be used for selective THz-photoexcitation of various types of nanostructures and nanoobjects, as well as molecules, especially in case if the excitation mechanisms are sensitive to both the polarisation and the amplitudes of the EM-field.

\section{Conclusion}

We have presented procedures capable of solving the problem of interaction of THz-range EMwaves with the metallic gratings having sub-wavelength periods. We have shown that the EMwave in the near-field zone under the metallic grating has a complicated vector structure, which is a result of superposition of the two fields, one of them being a field of dipoles with the charges induced at the edges of the metallic strips, and the second representing a field of the incident plane wave. It has been demonstrated that the total electric field of the transmitted wave has the two components along the directions parallel to the axis of grating and perpendicular to the grating plane. In the near-field zone, the amplitude ratio and the phase difference of these components vary depending on the local point under the grating. We have derived the time-averaged density of the electric energy for the spatial region under the grating. The effect of strong concentration of the electric energy of the THz wave near the edges of the metallic strips has been found. Some applications of the effects mentioned have been suggested for controlling THz-excitation of various nanostructures and nanodevices.

\section{Acknowledgement}

The authors would like to express their gratitude to Professor V. A. Kochelap and Professor A. E. Belyaev for their valuable discussions on various aspects of this work. 


\section{References}

1. Sakai K, Terahertz optoelectronics. Springer-Verlag: Berlin Heidelberg (2005).

2. Federici J and Moeller L, 2010. Review of terahertz and subterahertz wireless communications. J. Appl. Phys. 107: 111101.

3. Knap W, Kachorovskii V, Deng Y, Rumyantsev S, Lu J-Q, Gaska R, Shur M S, Simin G, $\mathrm{Hu}$ X, Asif Khan M, Saylor C A and Brunel L C, 2002. Nonresonant detection of terahertz radiation in field effect transistors. J. Appl. Phys. 91: 9346-9353.

4. Dyakonov M I, 2010. Generation and detection of terahertz radiation by field effect transistors. C. R. Physique. 11: 413-420.

5. Popov V V, Polishchuk O V and Shur M S, 2005. Resonant excitation of plasma oscillations in a partially gated two-dimensional electron layer. J. Appl. Phys. 98: 033510.

6. Aizin G, Popov V and Polishchuk O, 2007. Detection of terahertz radiation in a slit-grating gated field-effect transistor. Phys. Stat. Sol. C 4: 531-533.

7. Popov V V, 2011. Plasmon excitation and plasmonic detection of terahertz radiation in the grating-gate field-effect transistor structures. J. Infrared Milli Terahertz Waves. 32: 11781191.

8. Otsuji T, Karasawa H, Watanabe T, Suemitsu T, Suemitsu M, Sano E, Knap W and Ryzhii V, 2010. Emission of terahertz radiation from two-dimensional electron systems in semiconductor nano-heterostructures. C. R. Physique. 11: 421-432.

9. Laurent T, Sharma R, Torres J, Nouvel P, Blin S, Varani L, Cordier Y, Chmielowska M, Chenot S, Faurie J-P, Beaumont B, Shiktorov P, Starikov E, Gruzinskis V, Korotyeyev V V and Kochelap V A, 2011. Voltage-controlled sub-terahertz radiation transmission through GaN quantum well structure. Appl. Phys. Lett. 99: 082101.

10. Nogajewski K, Łusakowski J, Knap W, Popov V V, Teppe F, Rumyantsev S L and Shur M S, 2011. Localized and collective magnetoplasmon excitations in AlGaN/GaN-based gratinggate terahertz modulators, Appl. Phys. Lett. 99: 213501.

11. Mikhailov S A, 1999.Tunable solid-state far-infrared sources: New ideas and prospects. Appl. Phys. 2: 65-108.

12. Chan W L, Deibel J and Mittleman D M, 2007. Imaging with terahertz radiation, Rep. Prog. Phys. 70: 1325-1379.

13. Kawano $\mathrm{Y}$ and Ishibashi K, 2010. On-chip near-field terahertz detection based on a twodimensional electron gas. Physica E. 42: 1188-1191.

Lyaschuk Yu. M. and Korotyeyev V. V., 2012. Interaction of terahertz electromagnetic field with a metallic grating: Near-field zone. Ukr.J.Phys.Opt. 13: $142-150$.

Анотація. Побудовано теорію взаємодії ТГия-випромінювання з субхвильовою металічною граткою. Проведено дослідження структури електричного поля електромагнітної хвилі в близькій зоні иієї тратки. Одержано просторовий розподіл компонент вектора електричного поля та густини електричного енергії електромагнітної хвилі, щчо пройшла крізь тратку. Зазначено ефект істотного локального підсилення електричного поля. Проаналізовано просторові залежності поляризачї електромагнітної хвилі в близькій зоні тратки. 